University of Wollongong

Research Online

Faculty of Engineering and Information

Faculty of Engineering and Information

Sciences - Papers: Part A

Sciences

$1-1-2015$

\title{
A decentralized multiagent-based voltage control for catastrophic disturbances in a power system
}

Sk. Razibul Islam

University of Wollongong, sri703@uowmail.edu.au

Kashem M. Muttaqi

University of Wollongong, kashem@uow.edu.au

Danny Sutanto

University of Wollongong, soetanto@uow.edu.au

Follow this and additional works at: https://ro.uow.edu.au/eispapers

Part of the Engineering Commons, and the Science and Technology Studies Commons

Research Online is the open access institutional repository for the University of Wollongong. For further information contact the UOW Library: research-pubs@uow.edu.au 


\title{
A decentralized multiagent-based voltage control for catastrophic disturbances in a power system
}

\author{
Abstract \\ In this paper, a multiagent-based voltage and reactive power control in the case of a multiple contingency \\ is presented. Incorporating the agent-based autonomous feature to the intelligence of the remote terminal \\ units, the present power system control structure can be used to help in preventing system voltage \\ collapse during catastrophic disturbances. The control algorithm is based on a decentralized architecture \\ of intelligent agents and the determination of a local zone that can carry out quick countermeasures in a \\ decentralized manner as a multiagent system (MAS) during an emergency situation. An adaptive \\ determination of the local zones undergoing voltage collapse has been developed based on the electrical \\ distances among the generators and loads. Once assigned, the elements of the Jacobian matrix can be \\ used to determine the optimum actions that need to be carried out at each power system element (such \\ as increasing the voltages of generators and load shedding) within the assigned local zone. The contract \\ net protocol is used for agent interactions. Simulation results using the IEEE-57 bus system show that the \\ proposed method can act quickly to respond to emergency conditions to ensure that voltage collapse can \\ be avoided.

\section{Keywords} \\ power, decentralized, disturbances, catastrophic, control, voltage, system, multiagent

\section{Disciplines} \\ Engineering | Science and Technology Studies

\section{Publication Details} \\ S. Islam, K. M. Muttaqi \& D. Sutanto, "A decentralized multiagent-based voltage control for catastrophic \\ disturbances in a power system," IEEE Transactions on Industry Applications, vol. 51, (2) pp. 1201-1214, \\ 2015.
}




\title{
A Decentralized Multi-Agent based Voltage Control for Catastrophic Disturbances in a Power System
}

\author{
S. R. Islam, Student Member, IEEE, D. Sutanto, Senior Member, IEEE, and K. M. Muttaqi, Senior \\ Member, IEEE, Australian power Quality and Reliability Center, School of Electrical, Computer and \\ Telecommunications Engineering, University of Wollongong, New South Wales, Australia
}

\begin{abstract}
In this paper, a multi-agent based voltage and reactive power control in the case of a multiple contingency is presented. Incorporating the agent based autonomous feature to the intelligence of the Remote Terminal Units (RTUs), the present power system control structure can be used to help in preventing system voltage collapse during catastrophic disturbances. The control algorithm is based on a decentralized architecture of intelligent agents and the determination of a local zone that can carry out quick countermeasures in a decentralized manner as a multi-agent system (MAS) during an emergency situation. An adaptive determination of the local zones undergoing voltage collapse has been developed based on the electrical distances among the generators and loads. Once assigned, the elements of the Jacobian matrix can be used to determine the optimum actions that need to be carried out at each power system element (such as increasing the voltages of generators and load shedding) within the assigned local zone. The contract-net-protocol (CNP) is used for agent interactions. Simulation results using IEEE-57 bus system show that the proposed method can act quickly to respond to emergency conditions to ensure that voltage collapse can be avoided.
\end{abstract}

Index Terms-- Contract Net Protocol, Multi-Agent System, Reactive Power Control, Emergency Control.

\section{INTRODUCTION}

$\mathrm{P}$ OWER systems are normally designed to meet the forecasted annual peak demand and to provide secure operation in case of credible contingencies. This is provided by system reinforcement and protection systems to ensure that the power system operation is safe, stable, reliable and economical. Because of the low probability of multiple contingencies in a system, no automatic system protection is generally provided to safeguard the system against multiple contingencies [1]. However, many incidents of multiple contingencies have occurred worldwide, which had led to voltage collapse and widespread blackouts such as the events of July 2, 1996, August 10, 1996 [2], August 14, 2003 in Canada and North America [3], and November 4, 2006 in European Power System [4]. More recently, some blackout events have occurred on 16th January, 2007 in Victoria, Australia [5] and 30th July, 2012 in Northern India [6] which were caused by cascaded line failures that segregated the system into several islands. Therefore, a system-wide protection scheme is urgently needed to maintain the integrity of the transmission grid against such unpredictable multiple contingencies [7].

The phenomenon of voltage collapse is characterized by an initial slow stable phase lasting from several seconds to minutes after any disturbance followed by a sharp disruptive phase of voltage decline in the system [8]. The important findings from reported incidents of voltage collapse are [9]:

- The initial impact of a critical disturbance is in a limited region of the system.

- The short-term rotor over-excitation capacity offers a certain time period before abruptly collapsing.

- The affected region by the disturbance can be identified by the increase of excitation and reduction of voltage.

- The existing control system that provides safety of the individual equipment is not sufficient to provide control for the transmission grid.

- An automatic control strategy must be developed to mitigate the contingencies.

This paper describes a proposed decentralized multi-agent based voltage and reactive power control in the case of multiple contingencies to help in preventing system voltage instability characterized by a sudden decline in bus voltages and an increased amount of reactive generation in the surrounding area.

Multi-agent system (MAS) can facilitate self-organizations, self-steering and control paradigms with complex behavior even when the individual strategies of all their agents are simple. MAS has been applied in many fields of power engineering including fault diagnosis, network control, power system restoration, automation and market simulation [10]. MAS has also been applied for the management of demand responses and distributed storage in micro-grid [11], [13], a wide area current differential protection system [12], and a combined preventive and corrective power system emergency control [14].

Both centralized and decentralized coordination strategies using MAS have been reported in the literature [15]. However, a decentralized coordination strategy of intelligent agents is preferred to avoid the delay in transferring information to the central controller from the affected areas, performing calculation and receiving commands from the central controller. In this paper, a decentralized coordination strategy of local zones is proposed, where each local zone can make a quick autonomous decision to find the best solution for the power system following multiple contingencies to prevent voltage instability.

Many recent works have been reported in the literature for voltage control following system contingencies using MAS. A multi-agent collaboration protocol of secondary voltage controllers such as SVC and STATCOM to eliminate 
voltage violations in the pilot nodes has been proposed in [16]. The voltage controllers are treated as agents and a fuzzy logic learning algorithm has been used to train the agents. A similar approach using a different learning algorithm has also been proposed in [17] where the agents were trained by distributed reinforcement learning algorithm. Reference [18] used the contract net protocol to control the reactive power and voltage violation in case of a large disturbance. All these methods can provide voltage support to a certain extent depending on the reactive power capacity of the reactive power sources; however these papers have not taken into account the effect of insufficient reactive power capabilities and the need for load shedding.

Reference [19] proposed a multi-agent technique for both voltage and reactive power control to prevent voltage instability, where the primary bus voltage is controlled by 'reactive power control' and the secondary bus voltage is controlled by 'voltage control'. While the proposed method can maintain the voltages in the substations between the allowable ranges, the method does not take into account the generators' over-excitation and the subsequent exciter current limiter protection which can drive the system towards voltage instability. A multi-agent approach including emergency reactive power dispatch and load shedding has been proposed in [20]. The authors proposed a request-interaction protocol for VAR dispatch and contract-net-protocol for load shedding to control both the system voltage and generators' overexcitation in case of multiple contingencies. However, the author did not mention any strategy to optimize the VAR rescheduling and load shedding. A multi-agent based distribution system voltage control using contract-net-protocol has been proposed in [21], where an iterative negotiation between the agents was proposed to correct the voltage in the distribution feeder. The iterative negotiation will increase the time to find an optimum solution and hence it is not suitable for the application during system emergency, where time is of essence.

In this paper, a novel design of MAS using the existing SCADA based control system is proposed. The remote terminal units (RTUs), that can measure the electrical parameters such as voltage, current, power, frequency in the associated substations, will be used as intelligent agents. An adaptive determination of the local zones has been developed based on the electrical distances among the generators and loads. Initially, the network will be divided into local zones, where the load buses are grouped with the reactive power generating units, which are the closest to the load buses in terms of their electrical distances. Then the agents in each zone will work cooperatively to find the optimum control action to achieve an acceptable post-disturbance equilibrium condition. The multi-agent cooperative control protocol can coordinate a group of agents and achieve their group goals in real-time. The controls considered in this paper are varying the generator voltage reference setting and, as a last resort, intelligent applications of targeted load shedding. Reactive power sensitivity factors and voltage sensitivity factors to active and reactive power load have been formulated to determine the optimum amount of reactive power dispatch and load shedding.

The contribution of the paper is the novel adaptive determination of the local zone where the disturbances occur using electrical distances and the development of a multiagent decentralized control algorithm to determine the most optimum operation in the local zone to avoid voltage collapse.

\section{ZONE IDENTIFICATION AND ZONE FORMATION}

Since the effect of transmission line outages on the system is initially limited in a small zone, close to the point where contingency occurs, the power system, therefore, can be divided into local zones to utilize the limited geographical effect of the outage. These are the areas where the loads and the generators have sufficient electrical proximity so that when the system undergoes any critical disturbance, the actions of the controller in the affected zone can interpose prompt maneuver of the system towards the acceptable operating states and can have more impact on the voltage improvement.

The concept of electrical distance developed in [22] provides a good measure to identify different zones in the power system. Electrical distance is the impedance path between different nodes of the system and measures the relative voltage coupling. The concept of electrical distance is used in this paper to identify the different zones of voltage and reactive power control within the power system.

\section{A. Measures of Electrical Distance}

Electrical distance has been used in a number of power system problems [22]-[26]. There are a number of variant measures of electrical distance for a power network.

1) Sensitivity based method

The electrical distance can be quantified by the sensitivity matrix $\partial \mathbf{V} / \partial \mathbf{Q}$ which is the inverse of the matrix $\partial \mathbf{Q} / \partial \mathbf{V}$. $\partial \mathbf{Q} / \partial \mathbf{V}$ is part of the Jacobian matrix which appears during a load-flow computation following the Newton-Raphson method [22], [24]. In this approach, the electrical distance $\alpha_{\mathrm{ij}}$, is calculated as the attenuation of voltage variations between two nodes $i$ and $j$, given by

$$
\alpha_{i j}=\partial V_{i} / \partial V_{j}=\frac{\partial V_{i}}{\partial Q_{j}} / \frac{\partial V_{j}}{\partial Q_{j}}
$$

\section{2) Travelling wave based method}

The electrical distance has been calculated based on the time of energy transfer between two nodes in the system [25]. The difference between the phase angles of the travelling electromagnetic waves at these nodes is considered as the electrical distance.

3) Input impedance based method

The electrical distance has also been defined as the input impedance between two buses as:

$$
Z_{i j, \text { in }}=Z_{i i}+Z_{j j}-2 Z_{i j}
$$

where $Z_{i i}, Z_{j j}$ and $Z_{i j}$ are the elements of the bus impedance matrix.

4) Bus admittance matrix based method

One of the simplest methods is to use the absolute value of 
the inverse of the system admittance matrix [26]:

$$
[D]=\left|\left[Y_{B U S}\right]^{-1}\right|
$$

This distance matrix [D] with elements $d_{i j}$ gives the active and reactive power sensitivity with voltage changes between bus $i$ and $j$. The smaller the electrical distance, the higher the impact on the voltage change by the change in active and reactive power (for example due to a load shedding).

The elements of $\mathrm{Y}_{\mathrm{BUS}}$ are usually readily available, prior to the disturbances, from the control center, and as will be shown in the following section, the elements can be easily modified in case of contingency by the agents incorporating the system topology change into $Y_{\text {Bus. }}$ In this way, the proposed multi agent system can respond quickly from an earlier known admittance matrix. During the emergency condition, no global knowledge or the new $\mathrm{Y}_{\mathrm{BUS}}$ of the system is required; the agents will modify the $\mathrm{Y}$ matrix based on local information only. This method has been adopted in this paper for real time local zone identification.

\section{B. Defining Zones by Electrical Distance}

The objective of dividing the system into different local zones, each having intelligent agents, is to be able to represent each as MAS. Each MAS can cooperate with other local zones to find the best solutions for the problems by autonomous control of the voltage and reactive power in each local zone rather than waiting for the command from the central controller.

The performance of the local voltage control will depend on how the zones are determined. The zones can be determined by a bottom-up or agglomerate hierarchical clustering algorithm starting from the individual generator nodes and gradually encompassing the entire grid [24]. Another method is the K-means clustering that uses a topdown, or divisive approach which begins with a complete network, and then divides the network into clusters and finally adjusts those clusters based upon predefined criteria. The aim of the $\mathrm{K}$-means algorithm is to divide the $\mathrm{n}$ nodes in the network into $\mathrm{K}$ clusters so that the cluster distances are minimized [27].

Reactive power cannot be transmitted over long electrical distance [28], [29]. Therefore, it is necessary to form the cluster in such a way that any load in the cluster gets sufficient reactive power support from the system.

This requires that every local zone should include buses that can generate reactive power such as buses with generator, synchronous condenser, Static VAR compensator (SVC), and on load tap changers that can regulate voltage.

Hence, a zone is first defined such that the load buses are grouped with the reactive power generating units, which are closest to the load buses in terms of their electrical distances. This resembles the typical method of K-means clustering with the cluster centers fixed at the generator buses [27].

Initially, the zones will be identified for the base case system without any contingency. Let, $x_{i}$ represents a load bus at node $i$ in the system and $N_{G}$ is the number of generators/synchronous condensers. $S_{j}$ represents a zone where $j=\left\{1,2, \ldots . . N_{G}\right\}$, then $x_{i}$ is chosen to be in zone $S_{j}$ if the following criterion holds:

$$
S_{j}=\left\{x_{i}: d_{i j} \leq d_{i k} \forall 1 \leq k \leq N_{G}\right\}
$$

where $d_{i j}$ and $d_{i k}$ are the distances between the load $i$ and generators $j$ and $k$, respectively.

In this way, each load bus is grouped with its nearest generator and there will be $N_{G}$ zones in the system with one generator in each zone. After forming all the zones, if some generators have very few load buses or no load bus, then it is not realistic to keep them as separate zones. In this paper, a strategy has been made that if a zone has less than or equal to one load bus, we call it an ineffective zone. The electrical distance between the generator in the ineffective zone and the generators in the neighboring zones are compared. The lowest electrical distance is sought and the ineffective zone is merged into the neighboring zone corresponding to the lowest electrical distance. Thus, the zones are automatically formed for the pre-disturbance base case system.

\section{Zone Adaptation after Contingency}

Initially, the zones will be identified for the base case system without any contingency. Since the system topology will change after a contingency, such as due to transmission line outages, the electrical distances need to be recalculated using the modified bus admittance matrix $\left[Y^{\prime}\right]$. If there are $N$ buses in the system and $M$ transmission line outages, the modified matrix $\left[Y^{\prime}\right]$ can be calculated as:

$$
\left[Y^{\prime}\right]=[Y]-[M][\delta y][M]^{T}
$$

where [Y] is the original $N \times N$ admittance matrix, $[M]$ is a $N \times M$ connection matrix and $[\delta y]$ is a diagonal matrix containing the admittance of the outaged lines in the diagonal. Each column in $[M]$ corresponds to each line outage and contains +1 and -1 at the positions of the sending and receiving end, respectively. The rest of the values of $[M]$ are zero.

According to the Inverse Matrix Modification Lemma (IMML) [23], the inverse of [ $\left.Y^{\prime}\right]$ can be calculated as

$$
\left[Y^{\prime}\right]^{-1}=[Y]^{-1}-[Y]^{-1}[M][c][M]^{T}[Y]^{-1}
$$

where

$$
\begin{aligned}
& {[c]=\left([\delta y]^{-1}+[z]\right)^{-1}} \\
& {[z]=[M]^{T}[Y]^{-1}[M]}
\end{aligned}
$$

In this way, the electrical distance can be obtained quickly from the absolute value of the inverse of the modified system admittance matrix as given in (6) from the base case bus admittance matrix $[Y]$, which is usually available in advance, prior to the disturbance. No global knowledge of the system is required during the disturbance when applying this zone adaptation.

\section{DETERMINING OPTIMAL COUNTERMEASURES USING VOLTAGE SENSITIVITY APPROACH}

In order to develop a real time control of voltage instability, the voltage sensitivity method could be used to calculate the appropriate amount of countermeasures such as the increase in the generator voltage reference setting and the amount of load shedding [30]. The control algorithm should be able to determine the optimum value of the countermeasures to restore the load voltage magnitudes to a safe level within a reasonable time span and by a minimal amount of control 
actions.

In this paper, an attempt has been made to utilize the concept that the voltage increase in some selected generators/synchronous condensers would increase the load voltage magnitudes as well as relieve some of the generators whose reactive power have exceeded their reactive power limits [8]. In some cases, the reactive power outputs of these generators would be brought back below the maximum limit allowing them to participate in the control of the terminal voltages. The other control variable is load shedding which will come into action if the load voltages are not corrected by the action of generators' terminal voltage increment and the operation of the automatic OLTC within a pre-specified time limit.

\section{A. Varying the Generator Voltage Reference Setting}

Assuming that each zone does not have the voltage information of the global network, the voltage sensitivities with respect to the generators' reactive power outputs can be obtained from the decoupled load flow $Q-V$ equation [31] which can be written in matrix form as:

$$
[\Delta Q / V]=[B][\Delta V]
$$

where $[B]$ is the imaginary part of the bus admittance matrix.

The matrix given by (9) does not include the equations related to the generator buses in the traditional decoupled load flow formulation, because the voltages are specified for these buses. However in our proposed approach, the voltages of the generator buses will be varied to produce the necessary reactive power to reduce the reactive power deficit during post-contingency period. For this reason, the equations of the generator buses need to be included in (9). The generator buses and load buses can be separated where the matrix B can be partitioned into four sub matrices as follows:

$$
\left[\begin{array}{c}
\Delta Q_{G} / V_{G} \\
\Delta Q_{L} / V_{L}
\end{array}\right]=\left[\begin{array}{ll}
B_{G G} & B_{G L} \\
B_{L G} & B_{L L}
\end{array}\right]\left[\begin{array}{l}
\Delta V_{G} \\
\Delta V_{L}
\end{array}\right]
$$

where $\Delta Q_{G}$ (in MVAR) and $\Delta V_{G}$ (in pu ) correspond to the reactive power and voltage changes in the generator buses, respectively. $\Delta Q_{L}$ (in MVAR) and $\Delta V_{L}$ (in pu ) correspond to reactive power and voltage changes in the load buses, respectively. In the case of varying the generator voltage reference setting, the load is unchanged, i.e. $\Delta Q_{L}=0$ and equation (10) can be rewritten as:

$$
\left[\begin{array}{c}
\Delta Q_{G} / V_{G} \\
0
\end{array}\right]=\left[\begin{array}{ll}
B_{G G} & B_{G L} \\
B_{L G} & B_{L L}
\end{array}\right]\left[\begin{array}{l}
\Delta V_{G} \\
\Delta V_{L}
\end{array}\right]
$$

The incremental relationship between the change in the load voltage and the change in the generator voltage can be obtained from (11) assuming $B_{L L}$ is non-singular:

$$
\begin{gathered}
\Delta V_{L}=-B_{L L}^{-1} B_{L G} \Delta V_{G} \\
\text { from which: } \Delta Q_{G} / V_{G}=\left[B_{G G}-B_{G L} B_{L L}^{-1} B_{L G}\right] \Delta V_{G}
\end{gathered}
$$

Thus the load voltage sensitivity to the generator voltage change, denoted by $S_{\mathrm{LV}}$, is given by:

$$
S_{L V}=-B_{L L}^{-1} B_{L G}
$$

And the generator reactive power sensitivity to the generator voltage change, denoted by $\mathrm{S}_{\mathrm{QV}}$, is given by:

$$
S_{Q V}=\left(\operatorname{diag}\left[V_{G}\right]\right)\left[B_{G G}-B_{G L} B_{L L}^{-1} B_{L G}\right]
$$

After catastrophic disturbances, the load bus with the largest voltage drop will be selected as the target bus for the countermeasures. The load voltage sensitivity in (12) corresponding to the target bus will be used to find the generator bus that is most sensitive to the voltage change in the target bus. In this way, the voltage in the target bus can be improved by changing the voltage setting in the obtained generator bus. Once the most effective generator bus is found, and knowing the reactive power reserve (the reactive power limit minus the current reactive power output of the selected generator), the amount of voltage setting to be increased in the generator bus can be determined from (13), which should result in the increase of the target load bus voltage. As extra reactive power is injected into the system, all the other nodal voltages in the zone will also be improved.

\section{B. Load Shedding}

After the preliminary countermeasures of raising the terminal voltage of selected generators and synchronous condensers, the on-load tap changers are allowed to change automatically to try to improve the load voltages for a fixed period of time. This period of time is chosen in such a way that a margin of time is given prior to the operation of the first over-current limiter (the one with the lowest time to operate) in the rotor field circuit to limit the reactive power output of the generator, which has exceeded their reactive power limit that can lead to the onset of voltage instability to avoid cascading effect. If some load voltages are still below the lower limit at the end of the fixed period of time above, a strategic load shedding needs to be performed and the amount of load shedding can be calculated using the voltage sensitivity to active and reactive power load. Load shedding is a very effective mean of emergency voltage control if performed at right location, at the right time and at the right amount [32].

The decoupled load flow equations do not directly give the relationship between the voltage and the real power. Hence, to derive the load voltage sensitivity to active and reactive power load changes, the load flow equations are written in a rectangular form assuming a 'flat start' condition (all the load voltages are $1 \mathrm{pu}$. and angles are zero) as given in (16):

$$
\left[\begin{array}{c}
\Delta P \\
\Delta Q
\end{array}\right]=\left[\begin{array}{cc}
G & B \\
B & -G
\end{array}\right]\left[\begin{array}{l}
\Delta e \\
\Delta f
\end{array}\right]
$$

where $\Delta e$ (in pu) and $\Delta f$ (in pu) are the real and imaginary parts of the voltage difference, respectively. $G$ and $B$ are the real and imaginary parts of the bus admittance matrix, respectively. $\Delta P$ (in MW) and $\Delta Q$ (in MVAR) are the changes in active and reactive power load, respectively.

From (16), the voltage difference can be expressed in terms of real and reactive power as:

$$
\left[\begin{array}{c}
\Delta e \\
\Delta f
\end{array}\right]=\left[\begin{array}{ll}
S_{e P} & S_{e Q} \\
S_{f P} & S_{f Q}
\end{array}\right]\left[\begin{array}{l}
\Delta P \\
\Delta Q
\end{array}\right]
$$

where $S_{e P}, S_{e Q}, S_{f P}$ and $S_{f Q}$ are the sub-matrices that provide the sensitivities between voltage and power. $S_{e P}$ is the partial 
sensitivity of the real part of the voltage difference with respect to real power load, and similarly others. In the case of a load shedding at bus $k$, all the $\Delta P$ and $\Delta Q$ values at other nodes can be set to zero except for $\Delta P_{k}$ and $\Delta Q_{k}$. The change in $i$-th bus voltage magnitude due to load shedding at $k$-th bus can be obtained as:

$$
\Delta V_{i}=\sqrt{\left(\Delta e_{i}^{2}+\Delta f_{i}^{2}\right)}
$$

Using (17), (18) can be rewritten as:

$$
\Delta V_{i}=\sqrt{\begin{array}{l}
\left(S_{e P}(i, k) * \Delta P_{k}+S_{e Q}(i, k) * \Delta Q_{k}\right)^{2}+ \\
\left.\left(S_{f P}(i, k) * \Delta P_{k}+S_{f Q}(i, k) * \Delta Q_{k}\right)^{2}\right)
\end{array}}
$$

In the case of load shedding, the load power factor is assumed to be constant, and (19) can be rewritten as:

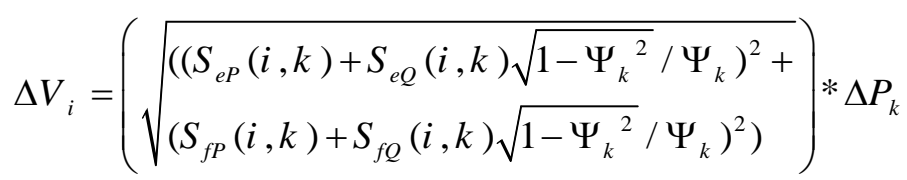

where the power factor at node $k$ is,

$$
\Psi_{k}=\frac{P_{k}}{\sqrt{P_{k}^{2}+Q_{k}^{2}}}
$$

Equation (20) can be re-written in the following form:

$$
\Delta V_{i}=S_{V L}(i, k) * \Delta P_{k}
$$

where the voltage sensitivity at bus $i$ to the active power (and implicitly voltage sensitivity to the reactive power) load shedding at bus $k$ is given by:

$$
S_{V L}(i, k)=\sqrt{\begin{array}{l}
\left(\left(S_{e P}(i, k)+S_{e Q}(i, k) \sqrt{1-\Psi_{k}^{2}} / \Psi_{k}\right)^{2}+\right. \\
\left.\left(S_{f P}(i, k)+S_{f Q}(i, k) \sqrt{1-\Psi_{k}^{2}} / \Psi_{k}\right)^{2}\right)
\end{array}}
$$

The load bus with the largest voltage drop after the fixed period of time specified is chosen as the target bus for load shedding. The load voltage sensitivity in (23) corresponding to the target bus will be used to find the load bus where the load shedding in that bus is most sensitive to the voltage change in the target bus. In this way, the voltage in the target bus can be best improved by shedding a minimal amount of load in the selected load bus. The amount of the desired voltage increase in the target bus can be determined from the difference between the lower limit of the target voltage bus and the current voltage value. Once the most effective load bus for the load shedding is found, the amount of load shedding in that bus can be determined from (20). The maximum amount of load available for load shedding in the selected load bus is the current load that can be interruptible in that bus. If the amount of load shedding calculated from (20) is less than the available interruptible load, then the desired voltage in the target bus can be obtained by applying the load shedding in the selected bus. Otherwise, the above procedure will be repeated until the desired voltage at the target bus is achieved by successively applying load shedding in the next sensitive buses.

\section{MAS-BASed Reactive Power And Voltage Control}

Modern power system is equipped with SCADA (Supervisory Control and Data Acquisition) that monitors and controls the entire system over a large area. The SCADA consists of a number of different devices communicating with each other, such as HMI (Human Machine Interface), MTU (Master Terminal Unit) and RTU (Remote Terminal Unit) [33]. A central MTU is located in the control center which communicates with the RTUs. The RTU is a composite device that collects signal from a sensor and converts the sensor signal to digital data and sends them to MTU. It is also responsible for executing instructions coming from the MTU. The accessibility of information among the RTUs has been made possible by direct communication between RTUs. A typical SCADA system architecture is shown in Fig. 1.

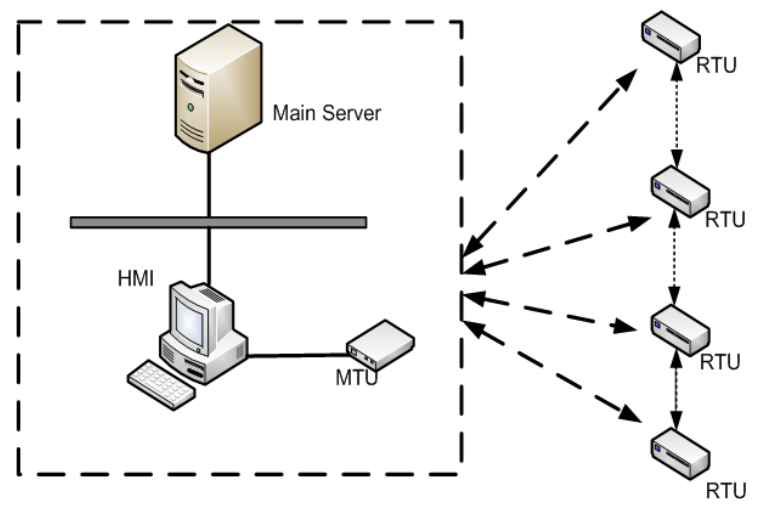

Fig. 1. SCADA system architecture

The main constraint in the SCADA based control system is that the RTUs are located far from the control center and in emergency the response from the control center may be too slow to direct necessary countermeasures in time to avoid potential voltage instability. For this reason, many of the modern RTUs are powerful enough to act as intelligent agents to autonomously monitor network parameters, communicate to other RTUs and make decisions without involving the host computers of the SCADA system.

The term 'intelligent agent' means an entity embedded with computer program that can automatically carry out some assigned tasks and can take autonomous decisions based on negotiation and any decision-making algorithm. An intelligent agent is an agent which exhibits proactivity (goal-directed behavior), social ability (ability to interact with other agents) and reactivity [34]. A hybrid reflex and goal-based model of intelligent agents with layered architecture has been adopted in this paper.

\section{A. Proposed MAS Layers}

The multi-agent system proposed for emergency voltage and reactive power control has two layers: Reactive Layer and Deliberative Layer and follows a vertical layered structure [34] as shown in Fig. 2.

Two types of intelligent agents have been considered for the proposed voltage/reactive power emergency control: Generator Agent (GA) and Load Agent (LA). The LAs in the reactive layer are modelled as simple reflex agents [35]. The agent function is based on some pre-defined condition-action 
rules i.e. if load voltage below minimum limit then send REQUEST message to GA etc. When a critical contingency that produces violations in the load voltage magnitudes occurs in the system, the deliberative layer becomes active. Both GAs and LAs work in this layer to systematically remove the load voltage violations through negotiation and based on the sensitivity model of the system. The GAs exhibits modelbased goal-oriented behavior [35]. The goal is to improve the load voltages above the minimum admissible limit with minimal amount of load shedding.

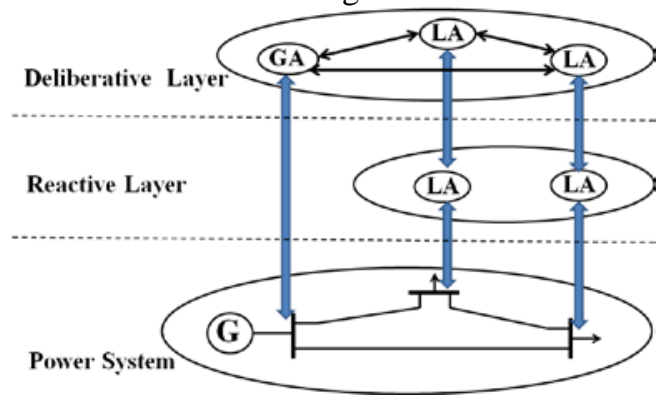

Fig. 2. Layered structure of the proposed MAS

Fig. 3 shows the functional diagram of the agent based RTU in the MAS environment.

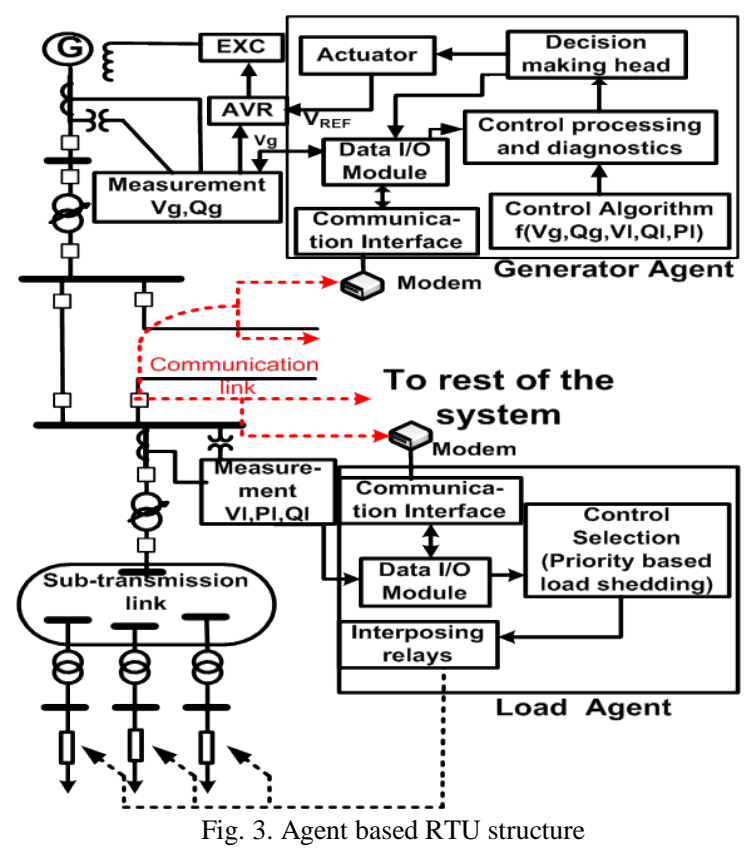

The agents within the RTU perceive the environment through sensors and act upon it through the actuators. The inputs to the sensor are the local electrical parameters such as voltage, current, tap position, breaker status, etc. A two-way communication link among the RTUs provides the message transfer capability for the agent interaction. Decision is made based on the local measurement as well as the information received from other agents.

The GA takes the measurements of voltage and reactive power from the system and sends it to the control processing unit. The control processing unit also gets the messages from other agents through the communication interface. GA takes the necessary decision on the adjustment of the generator's terminal voltage based on the control algorithm and implements it through the actuator by changing the AVR reference voltage. The decision of load shedding is implemented by LA which also works in a similar fashion. It applies load shedding to the associated bus by opening the circuit breaker in the feeder through the interposing relay operation.

\section{B. MAS Control Strategy and Agent Co-ordination}

The Foundation of Intelligent Physical Agent (FIPA) has developed certain interaction protocols using a standard set of communicative act with a well-defined semantics [36]. A widely accepted task sharing protocol in multi-agent system is the Contract Net Interaction Protocol (CNP) [37].

In this protocol, each agent is represented as a manager or a contractor. When an agent realizes that it cannot solve the present task by itself, it announces the task to other agents in the system and act as a manager of that task. An agent that receives the announcement will decide whether it is capable of carrying out the task and if so submits a bid for the task as a contractor. The manager agent then receives the bids from the potential contractors and decides who should be awarded the contracts in order to achieve an optimal solution of the task. The contract awards are then communicated to the agents that have submitted the bids. The winning contractors then take the initiative to fulfil the assigned task. An agent can be simultaneously a manager and a contractor for different tasks. The negotiation process during the CNP is shown in Fig. 4.

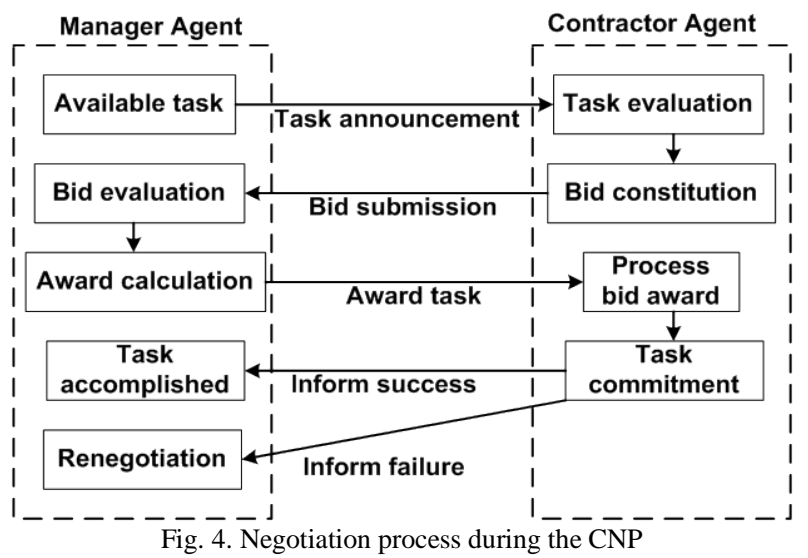

In the proposed multi-agent based emergency control system, the contract-net-protocol will be used for agent interaction. The GA can act both as a manager and a contractor, where as the LA will act as a contractor only. The step by step procedure of the negotiation strategy is given as follows:

Step 1: After a contingency has been identified in the system, the LA at each of the terminals of the outaged line broadcasts a message informing the event to all the agents. The agents that receive the message update their electrical distances and subscribe to their nearest generator as described in section II. In this way, GAs obtain the information of the modified zone.

Algorithm: Zone Forming Algorithm

Input: Load Agents (LA), Generator Agents (GA)

Output: Zones

for each $L A \alpha$ do for each $G A \beta$ do 


$$
\begin{aligned}
& \text { Calculate } d_{\alpha \beta} ; \\
& \beta_{\text {min }}=\underset{\beta}{\arg \min } d_{\alpha \beta} ; \\
& \text { Zone }_{\beta \min }=\text { Zone }_{\beta \min } \cup\{\alpha\} ;
\end{aligned}
$$

end

The LAs that find their load voltages lower than the specified limit inform the GA the magnitude of the voltages and request for voltage support. The GA, after knowing the load voltage magnitudes in the zone, selects the load bus with maximum voltage deviation from the reference value as the target bus for the control actions.

Step 2: The GA in the violated voltage zone specifies a task of reactive power support issuing a call for proposal (CFP) to other GAs in the system and acts as a manager GA. The GAs that receive the message, inform the manager GA of their available reactive power reserves and the terminal voltages. The manager GA after receiving all the bids from the GAs, or after the deadline, will calculate the amount of reactive support for the potential contractors. This will be assigned as follows:

The generator $i$ with the highest voltage sensitivity factor, $S_{L V}(t g, i)$ to the target bus voltage and with a positive reactive power reserve will be chosen first to dispatch. The amount of reactive power increase $\Delta Q_{G i}$ can be calculated as:

$$
\Delta Q_{G i}=\min \left[\frac{S_{Q V}(i, i)}{S_{L V}(t g, i)} *\left(V_{t g}^{\min }-V_{t g}\right), \Delta Q_{R i}, S_{Q V}(i, i) *\left(V_{G i}^{\max }-V_{G i}\right)\right]
$$

where $V_{t g}$ and $V_{t g}^{\min }$ are the current voltage and minimum operating voltage of the target bus respectively, $\Delta Q_{R i}$ is the reactive power reserve of the $i$-th generator $V_{G i}$ and $V_{G i}^{\max }$ are the current terminal voltage and maximum terminal voltage of the $i$-th generator, respectively. If the amount of reactive power is not sufficient to raise the target bus voltage to the desired value, the reactive power reserves of the generators are updated as:

$$
\left.\Delta Q_{R j}(\text { new })=\Delta Q_{R j} \text { (old }\right)+S_{G V}(i, j) * \Delta V_{G i}, \quad j \in N_{G}
$$

where $\Delta Q_{R j}($ old $)$ is the previous reactive power reserve and $\Delta Q_{R j}($ new) is the updated reactive power reserve. The generator with the highest value of the sensitivity factor and with a positive reactive power reserve is selected again as the next candidate to increase the reactive generation. The process is repeated until the desired voltage support at the target bus is achieved or the limit constraints are met. The manager GA then sends an accept-proposal act to the contractor GAs to increase the terminal voltage of the generator by the specified amount. The process of the optimal reactive power dispatch is shown in the flow diagram in Fig. 5.

Step 3: After completing the reactive power scheduling task, the manager GA waits for a fixed period of times to allow other normal voltage control actions to operate, such as switched capacitors, OLTC, etc. If the target bus voltage does not come within the limit by the end of the fixed period, the GA initiates the load shedding procedure. The GA sends a call for proposal (CFP) to the LAs in the zone. The LAs reply with their load voltages and load active and reactive powers. The amount of load shedding is calculated following the same procedure described in step 2 .
First, the load bus $i$ with the highest value of sensitivity $S_{V L}(t g, i)$ is selected to shed the load. The load shedding amount $\Delta P_{L}$ is calculated as:

$$
\Delta P_{L}=\min \left(P_{L i}, \frac{V_{t g}^{R E F}-V_{t g}}{S_{V L}(t g, i)}\right)
$$

where $P_{L i}$ is the current load of bus $i$.

If the specified load shed at bus i does not bring the target voltage over the minimum limit, the load bus with the second highest value of sensitivity is selected for further load shedding. This continues until the target bus voltage come within the limit.

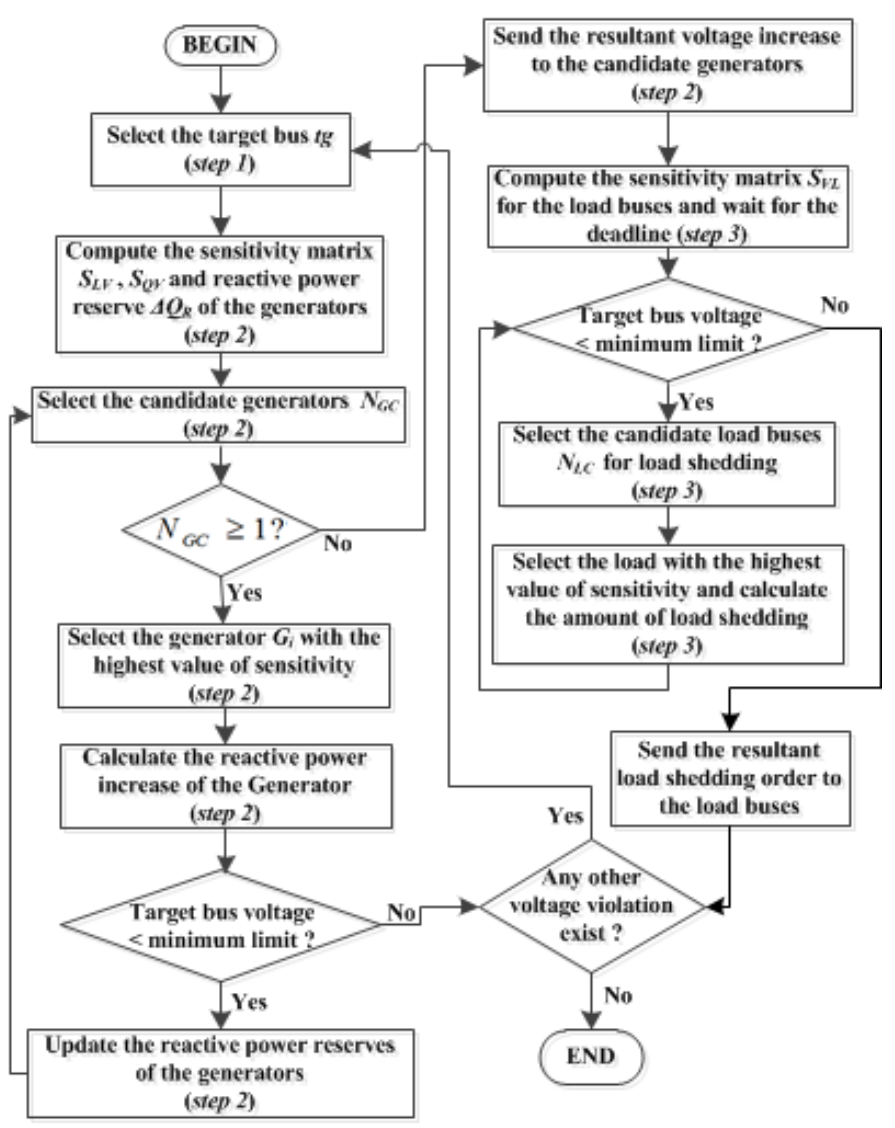

Fig. 5. Flow diagram of the control strategy

The amounts of load shedding so calculated will be sent to the respective LAs. These LAs, after receiving this information, will curtail the loads by successively opening the distribution feeder until the loads are shed by the desired amount.

The proposed multi-agent system is different to that described in [21], as the communications between the generator and load agents are assigned in a single time step rather than iteratively as suggested in [21]. This reduces the communication overhead between the agents. After the target bus voltage has been controlled to be within the limit, the GA checks whether there is any other voltage violation in the zone or not. The process is repeated until all the voltages come within allowable limits as shown in Fig. 5. 


\section{Design and Implementation of the Proposed MAS}

The proposed MAS has been implemented using Java Agent Development Framework (JADE) [34]. JADE is a FIPA compliant open source agent simulation software with wellspecified semantics for agent communication. It is implemented in Java programming language and works as a middleware for the development and run-time execution of peer-to-peer applications that use agents. The negotiation among the agents in JADE is performed through interchanging messages which use FIPA-specified Agent Communication Language (ACL). The ACL messages passed among the agents are characterized by (i) performative (ii) conversation ID (iii) sender (iv) intended receiver and (v) content.

In order to fulfill the task of decentralized emergency voltage control, the agents need to communicate with each other to exchange information of bus voltages and generator reactive powers. This information is shared among the agents through transmission of messages with pre-defined templates. Table I shows the required information of the agents both in normal and emergency states in order take part in the control mechanism and negotiation. This work is done within the agent behaviors. In this paper, we have defined five userspecified agent behaviors; each of them is the extension of agent's cyclic behavior. Table II shows the performative, conversation ID, content and sender/receivers of messages associated with the behaviors of the agents.

TABLE I

REQUIRED INFORMATION OF THE AGENTS

\begin{tabular}{|l|l|l|}
\hline & Normal State & Emergency State \\
\hline $\begin{array}{l}\text { Generator } \\
\text { Agent }\end{array}$ & $\begin{array}{l}\text { Bus } \\
\text { admittance } \\
\text { matrix }\end{array}$ & $\begin{array}{l}\text { Load voltages } \\
\text { Generator voltages } \\
\text { Generator reactive powers } \\
\text { Load power consumption } \\
\text { (active and reactive) } \\
\text { ID of the outaged line(s) }\end{array}$ \\
\hline Load Agent & $\begin{array}{l}\text { Load voltage } \\
\text { Breaker status }\end{array}$ & $\begin{array}{l}\text { Load voltage } \\
\text { Load power consumption } \\
\text { (active and reactive) }\end{array}$ \\
\hline
\end{tabular}

\section{1) Update Electrical Distance}

This behavior is implemented in step 1 of section IV. On the event of a line outage, the LA/GA nearest to the outaged line sends an INFORM message with conversation ID "Elec_Dis" to all other agents. The content of this message is "type, name, outaged bus number". Type indicates whether it is from load agent or generator agent, name is the local name of the sending agent and outaged bus number is the sending/receiving end bus number of the outaged line. With this information, the agents can update the electrical distance as described in section II (C).

2) Update Zone

This behavior also corresponds to step 1 of section IV. After updating the electrical distance, the LAs send an INFORM message with conversation ID "Zone" to a GA to register with this agent. This GA has the lowest electrical distance with the sending LAs among all other GAs.

\section{3) Need Voltage Support}

When a LA detects a violation in voltage, it sends a message to the GA with performative "REQUEST", conversation ID "Voltage Support" and content as "type, name, bus voltage", as described in step 1 of section IV.

4) Increase Reactive Power

The CNP for generator reactive power increase is implemented in this behavior. Four types of messages are associated with this behavior. The explanations of the messages are given in step 2 of section IV.

\section{5) Load Shedding}

The CNP for load shedding is implemented in this behavior. Four types of messages are associated with this behavior. The explanations of the messages are given in step 3 of section IV-B.

During the emergency condition, the whole process is totally autonomous carried out by the agents.

TABLE II

Agent BeHavior For the Proposed MAS

\begin{tabular}{|c|c|}
\hline Behaviour & Message Template \\
\hline $\begin{array}{l}\text { Update Electrical } \\
\text { Distance }\end{array}$ & $\begin{array}{l}\text { Performative "INFORM", ID "Elec_Dis", sender "Outaged } \\
\text { line LA/GA", receiver "LA/GA", content "type, name, } \\
\text { outaged bus number" }\end{array}$ \\
\hline Update Zone & $\begin{array}{l}\text { Performative "INFORM", ID "Zone", sender "LA", } \\
\text { receiver "GA", content "type, name" }\end{array}$ \\
\hline $\begin{array}{l}\text { Need Voltage } \\
\text { Support }\end{array}$ & $\begin{array}{l}\text { Performative "REQUET", ID "Voltage_Support", sender" } \\
\text { LA", receiver "GA", content "type, name, bus voltage" }\end{array}$ \\
\hline \multirow[t]{4}{*}{$\begin{array}{l}\text { Increase Reactive } \\
\text { Power }\end{array}$} & $\begin{array}{l}\text { Performative "CFP", ID "Reactive_Power", sender " } \\
\text { manager GA", receiver "GA", content " " }\end{array}$ \\
\hline & $\begin{array}{l}\text { Performative "PROPOSE", ID "Reactive_Power", sender " } \\
\text { GA", receiver "manager GA", content "bus no., bus voltage, } \\
\text { reactive power reserve" }\end{array}$ \\
\hline & $\begin{array}{l}\text { Performative "ACCEPT_PROPOSAL", ID "Reactive_ } \\
\text { Power", sender "manager GA", receiver "GA", content } \\
\text { "voltage change" }\end{array}$ \\
\hline & $\begin{array}{l}\text { Performative "INFORMFAILURE", ID "Reactive } \\
\text { Power", sender "GA", receiver "manager GA", content " " }\end{array}$ \\
\hline \multirow[t]{4}{*}{ Load Shedding } & $\begin{array}{l}\text { Performative "CFP", ID "Load_Shedding", sender " } \\
\text { manager GA", receiver "LA", content " }\end{array}$ \\
\hline & $\begin{array}{l}\text { Performative "PROPOSE", ID "Load_Shedding", sender " } \\
\text { LA", receiver "manager GA", content "bus no., active } \\
\text { power, reactive power" }\end{array}$ \\
\hline & $\begin{array}{l}\text { Performative "ACCEPT_PROPOSAL", ID } \\
\text { "Load_Shedding", sender " manager GA", receiver "LA", } \\
\text { content "load power change" }\end{array}$ \\
\hline & $\begin{array}{l}\text { Performative "INFORMFAILURE", ID "Load_Shedding", } \\
\text { sender "LA", receiver "manager GA", content " " }\end{array}$ \\
\hline
\end{tabular}

\section{TEST RESUltS AND DISCUSSIONS}

In order to evaluate the effectiveness of the proposed MAS based emergency control scheme, the IEEE-57 test system [38] shown in Fig. 6 has been simulated using PSAT [39] to carry out the proposed emergency reactive power and voltage control.

The IEEE-57 test system has seven synchronous machines, each of which is modeled by a six order machine model including the type II Automatic Voltage Regulator (AVR) and over-excitation limiter (OXL) model. Initially, the electrical distances of all the buses prior to the contingency are calculated using (7) and the zones are defined using the clustering approach given by (11). Each load bus is grouped with its closest generator in terms of electrical distance as shown in Fig. 6(a). However, the generators at bus 1, 2 and 6 are in the ineffective zones. Therefore, they are merged into the neighboring zones according to the principle described in section II(B) and finally four zones are formed for the predisturbance base case system, as shown in Fig. 6(b). 


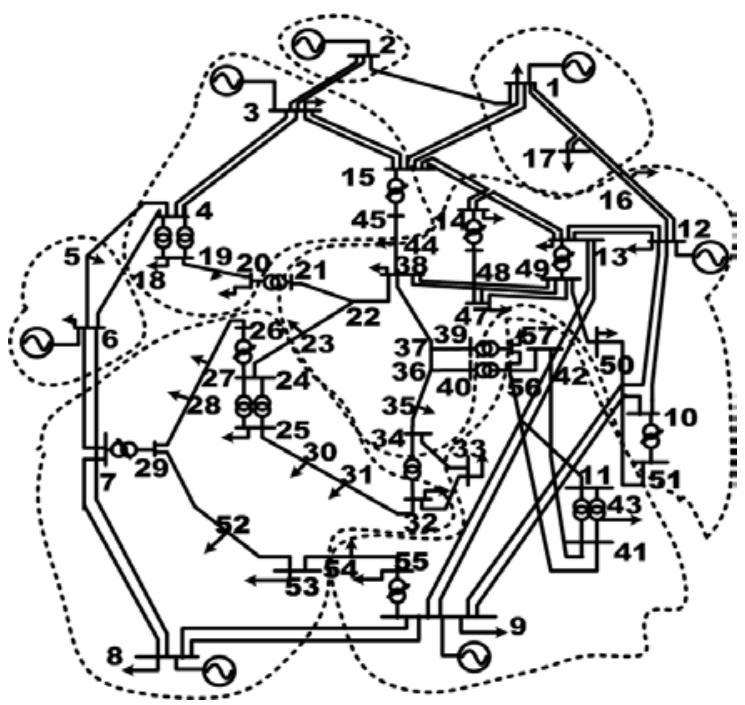

(a)

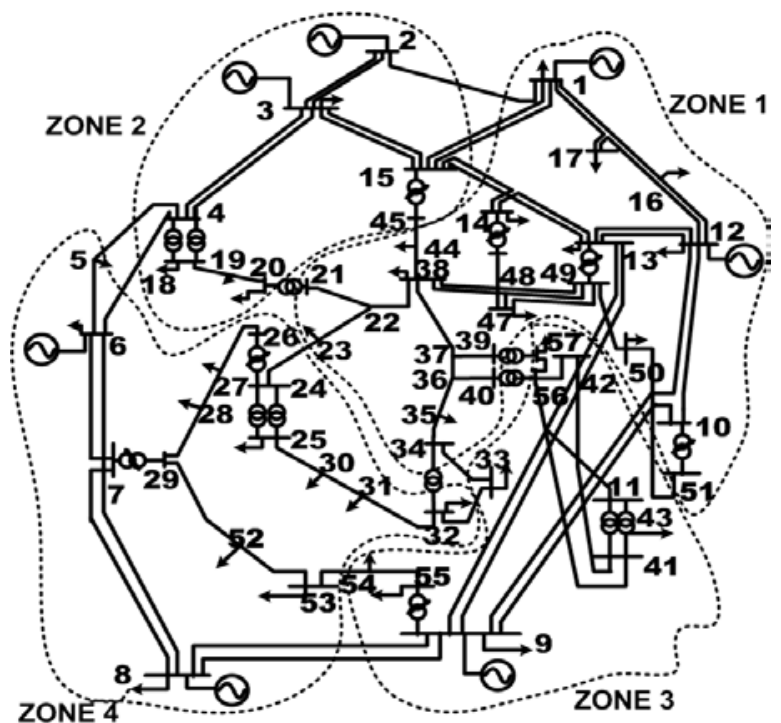

(b)

Fig. 6. IEEE 57 bus test system: (a) Initial zones of the system without adjustment (b) Initial zones of the system after adjustment.

The agents in JADE can read/write the power system data via Transmission Control Protocol (TCP/IP) communication through MATLAB Instrument Control Toolbox [40]. As shown in Fig. 7, the TCP_Agent in JADE collects the snapshot of the load voltages and generator reactive powers from PSAT at each control instance and transmits the data to the relevant agents. The required sensitivities for optimal control actions are computed by calling MATLAB from JAVA. The control actions resulted from the negotiation among the agents are then passed back to the TCP_Agent; which transfers these data again to PSAT.

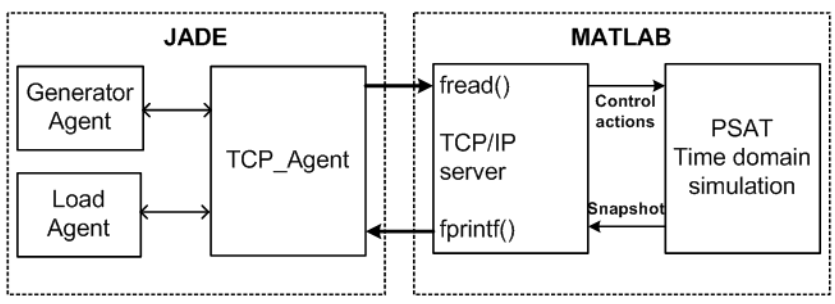

Fig. 7. Data exchange between MATLAB and JADE

\section{A. Case 1: Line Outage of 36-37 and 37-38}

The loss of lines $36-37$ and $37-38$ is simulated to test the proposed emergency reactive power and voltage controller. This has resulted in changes to electrical distances and required the re-zoning of some of the buses as shown in Fig. 8. The voltage profile of all buses prior and after the disturbances is shown in Figure 9. Figure 9 shows that the lowest voltage after the disturbance is at node 34 .

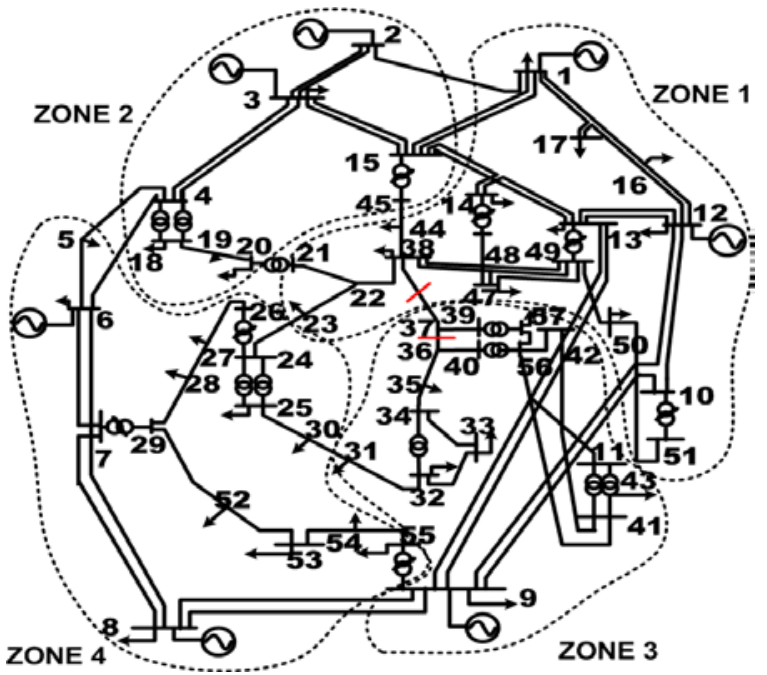

Fig. 8. Modified zones of the system after the contingency in case 1.

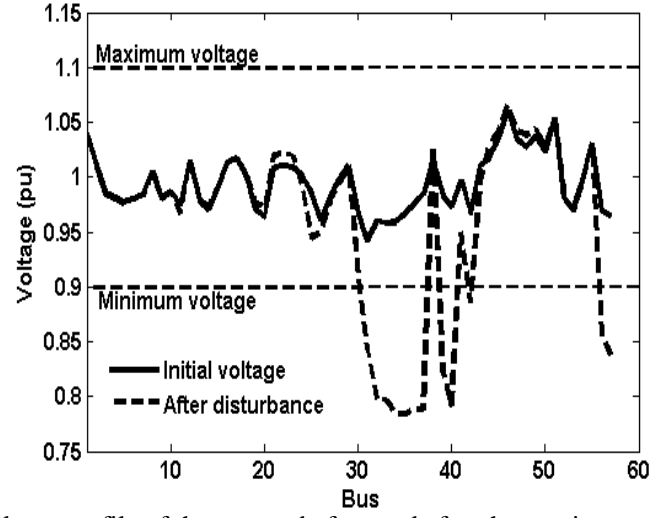

Fig. 9. Voltage profile of the system before and after the contingency

\section{B. Reactive Power Dispatch under Emergency}

When any of the load voltage drops below a pre-specified limit, the emergency reactive power dispatch is activated. It is recommended to wait until transients have settled down and the line auto-reclosure time is exceeded. To allow this, the agents will start the negotiation process after $10 \mathrm{sec}$, if the voltage violation still occurs. During the $10 \mathrm{sec}$ period, the LAs update the electrical distances and subscribe to the nearest generator to set up the zones, each of which can act like a MAS. In this case, only the generator at bus 9 (GA 9) has exceeded the maximum reactive power and the load voltages that have gone below $0.9 \mathrm{pu}$ are also in zone 3 . As a result, the countermeasures will be initiated only in zone 3 . The load agents having bus voltages below 0.9 pu send request message to GA 9 in zone 3 for voltage support. The GA 9 in zone 3 finds the maximum voltage deviation at bus 34 and sets this bus as the target bus for the control actions. At first, GA 9 initiates the CNP for reactive power dispatch and sends a CFP to other generators. 
It is anticipated that the deadline for sending the proposals is short enough to ensure quick responses from the generators. As a result, not all the generators in the system will be able to respond due to communication delay. But that does not hamper the control strategy because only the generators in the surrounding regions will have significant impact on the voltage improvement of the affected buses. Let us assume that only generators 8 and 12 have been able to respond to the CFP within the deadline. Therefore, only generators 8,9 and 12 will be considered for the reactive power dispatch. GA 8 and GA 12 respond with their bids given below:

GA 8: (1.005, 64.096, 200) GA 12: $(1.015,129.71 ., 155)$

The figures in the bids correspond to each generator's terminal voltage (in pu), current reactive power generation and maximum Q limit (in MVAR), respectively. GA 9 knows its own generator's terminal voltage, the Q-output and the Q-limit which are $0.97981 \mathrm{pu}, 13.43$ MVAR and 9 MVAR, respectively. Once GA 9 gets these values, it calculates the amount of voltage increase for the candidate generators and sends these dispatch awards to the agents which are:

GA 8: 0.0815 pu., GA 9: 0.0578 pu., GA 12: 0.0398 pu. Notice that GA 9 also increases its terminal voltage although initially its $\mathrm{Q}$ output was over the maximum limit. This is because the other two generators have increased the reactive power generation resulting in GA 9 reactive power to go below its reactive power limit and hence the terminal voltage of GA 9 is allowed to be increased. Once the GAs receive their contracts, they increase their voltages accordingly by increasing the AVR reference voltages.

\section{Load Shedding Under Emergency}

In this case, the deadline for load shedding is considered to be 30 seconds i.e. after 30 seconds of the disturbance, if the voltages and reactive powers are not within limits, the GA will start the load shedding procedure. After 30 seconds, the lowest bus voltage is found to be 0.78759 pu at bus 34 . As a result, GA 9 selects this bus as a target bus and starts the load shedding procedure. GA sends another CFP to the LAs in the zone. The LAs reply with the current voltage and power. Then GA 9 starts the process of load shedding. The solution converges with $6 \mathrm{MW}$ load shedding at bus 35 and $2.97 \mathrm{MW}$ load shedding at bus 33 . After applying the specified amount of load shedding, the target bus voltage is found to be 0.90982 $\mathrm{pu}$, which is within the limit and no other voltage violation exists. So, a solution has been obtained and therefore MAS stops the control process. The improvement in the load bus voltages and the generator reactive powers are shown in Fig. 10 and the voltage profiles at different stages are shown in Fig. 11.

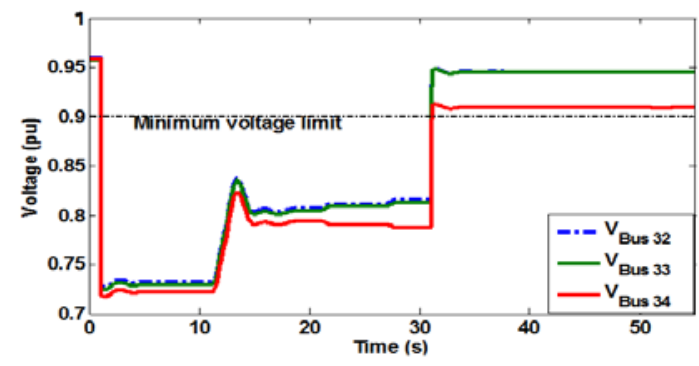

(a)

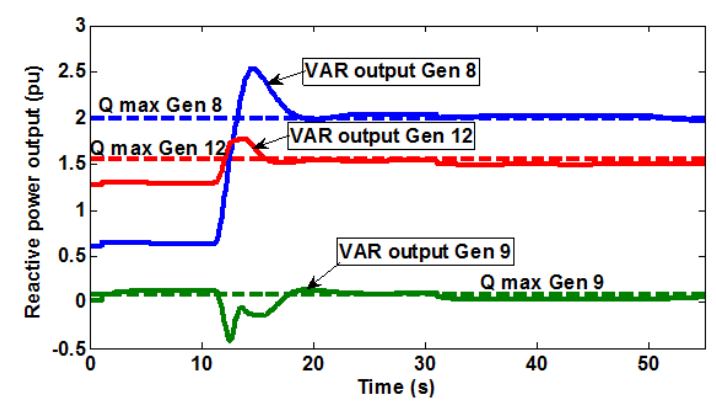

(b)

Fig. 10. The bus voltages (a) and reactive power (b) change for contingency in case 1.

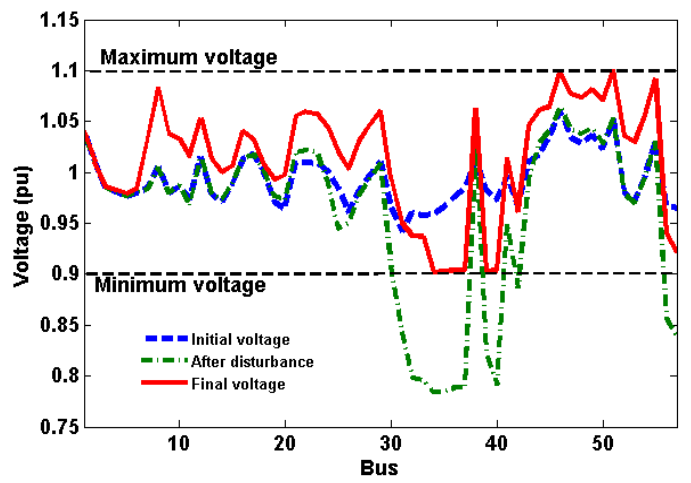

Fig. 11. Voltage profile at different stages for contingency in case 1.

\section{Case 2: Line Outage of 31-32 and 32-34}

Before applying this contingency, the system load was increased by 20 percent except for those buses where load increase causes voltage violation. This case has been selected to show the effectiveness and performance of the proposed MAS based control strategy in the case of more than one zone is affected. After applying the contingency, the zones are modified according to the electrical distance which is shown in Fig.12.

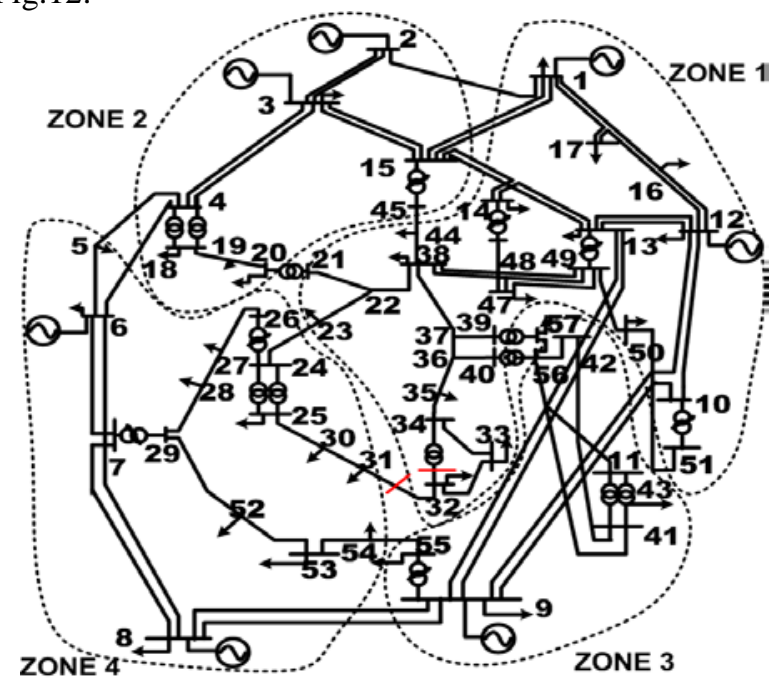

Fig. 12. Modified zones for the contingency in case 2

In this case, both zones 1 and 4 are affected and the target buses for these zones are bus $31(0.83792 \mathrm{pu})$ and bus 32 $(0.8594 \mathrm{pu})$, respectively. So, GA 8 and GA 12 start the control procedure and send CFP for generator reactive power scheduling. Assuming that GA 6 responds to GA 8, and GA 1 responds to GA 12, the submitted bids for these generators are: 
GA 1: (1.04, 141.26, 200)

GA 6: $(0.98,14.98,25)$

The similar values of terminal voltages and reactive powers of GA 8 and GA 12 are:

GA 8: $(1.005,86.5,200)$

GA 12: (1.0093, 155.6, 155)

The calculated voltage increases for these generators are:

GA 1: 0.0129 pu., GA 6: 0.0322 pu.

GA 8: $0.0509 \mathrm{pu}$., GA 12: $0.0104 \mathrm{pu}$.

At $30 \mathrm{sec}$, the target bus voltages are still below $0.9 \mathrm{pu}$ (0.86224 pu at bus 31 and 0.87273 pu at bus 32). As a result, GA 8 and GA 12 start the load shedding procedure in their zones, namely zone 4 and zone 1 , respectively. In this case, the amount of load shedding as calculated by the manager agents are $2.6 \mathrm{MW}$ at bus 31 in zone 4 and $2 \mathrm{MW}$ at bus 32 in zone 1 . When the LA 31 and LA 32 shed the specified amount of load, the voltages at these buses rise to $0.9006 \mathrm{pu}$ and $0.8998 \mathrm{pu}$, respectively. Since these values are within the tolerance limit of $0.001 \mathrm{pu}$, the solution is accepted. All the load bus voltages are within the acceptable limits (0.9-1.1 pu) as shown in Fig. 13 and the agents stop the control procedure.

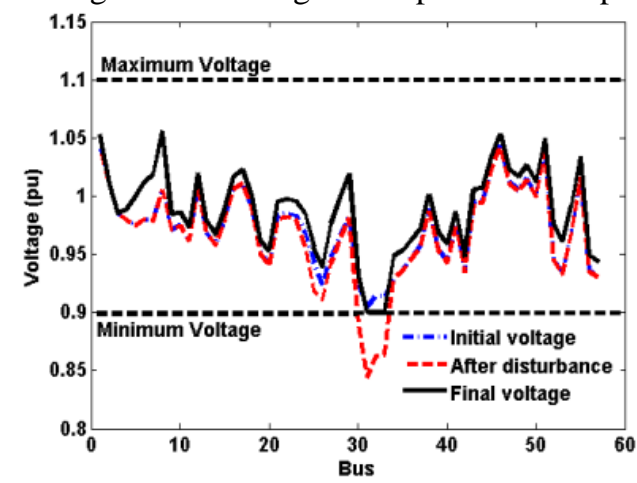

Fig. 13. Voltage profile at different contingency in case 2.

Fig. 14 shows the load bus voltage magnitudes and the changes in the reactive power outputs of the generators. It can be observed that the reactive power outputs of all the generators involved have been increased to their maximum limits and the load shedding at $30 \mathrm{sec}$ has resulted in the voltages at the target bus voltage (bus 31 and 32) magnitudes to be within the tolerance of the limits specified.

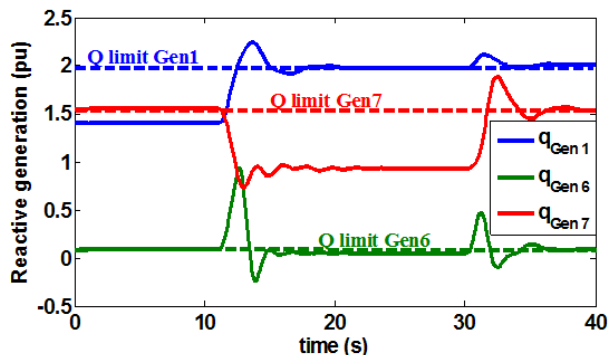

(a)

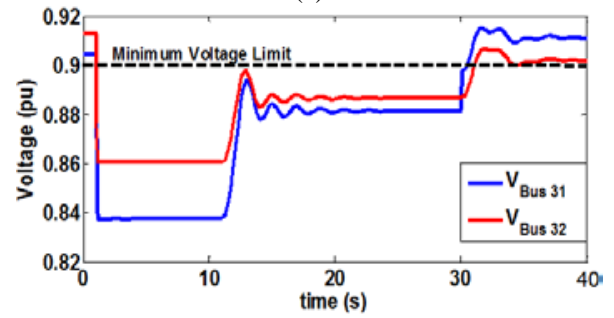

(b)

Fig. 14. Change in load voltage (a) and reactive power outputs (b) of the generators for contingency in case 2 .

\section{E. Case 3: Effect of Communication and Implementation Delay}

The proposed MAS based emergency voltage control scheme might introduce a delay in implementing the actions because of the communication among the agents. In particular, the load shedding will be performed by direct tripping the load from the utility transmission sub-station through under-voltage relay installed at the primary of the distribution sub-station located close to key transmission sub-stations [41]. This would also cause additional delay in actually shedding the loads. Fig. 15 shows the delay between the detection of voltage violation and the actual implementation of the countermeasures on a time scale. The total delay $T_{\text {delay }}$ can be expressed as

$$
T_{\text {delay }}=t_{\text {neg }}+t_{\text {com }}+t_{\text {imp }}
$$

where $t_{\text {neg }}$ is the time required by the agents for negotiation which includes the communication delay among the agents, $t_{\text {com }}$ is the time for computation of the sensitivities and algorithm and $t_{i m p}$ is the time to implement the actions after decision making.

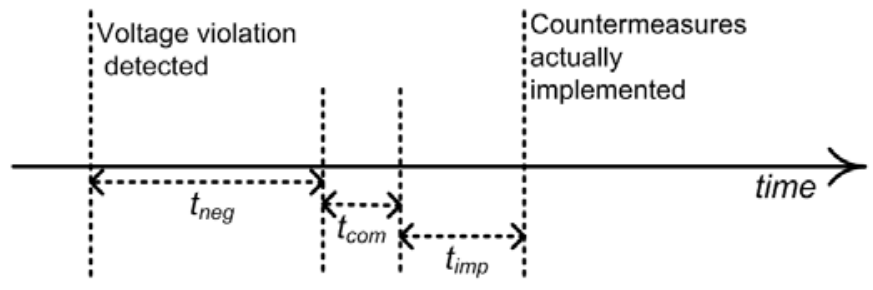

Fig. 15. Delay time between the occurrence of voltage violation

Long term voltage instability scenario is typically monotonic [8] i.e. the voltage decays slowly over a period of minute or more before abruptly collapsing. Based on this assumption, one can expect that the countermeasures can be successfully implemented with the above mentioned delays without causing any significant deviation in the response. To illustrate this, we have considered 10 seconds delay between detection and implementation by the proposed MAS for the scenario described in case 1 . Fig. 16 shows the voltage at bus 34 in case 1. For comparison, the response without delay is shown in dotted line. It can be seen that the countermeasures can successfully stabilize the system.

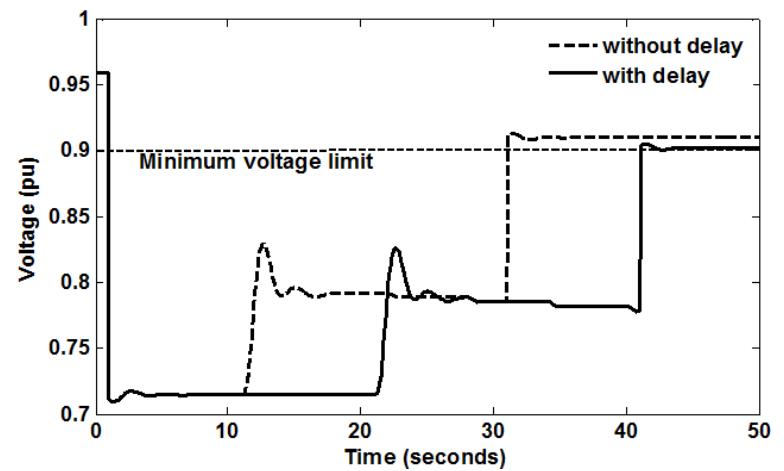

Fig. 16. Voltage at bus 34 in case 1 with and without considering delay.

The actual delay in the response of the proposed MAS will depend on the communication facility in the transmission system and between the RTU and IED (Intelligent Electronic Device) relay that will trip the distribution feeder. The wide- 
area network based on high speed optical fiber network with 155.52 Mbps can facilitate to communicate over $180 \mathrm{~km}$ distance with $1.3 \mathrm{~ms}$ delay time [42].With the extensive deployment of substation automation, Ethernet based local area network can be applied for communication between RTU and IED relay. According to IEEE standard 802.3, for an Ethernet with a maximum of $2.5 \mathrm{~km}$ in length and four repeaters, the maximum transmit delay should not exceed 25.6 $\mu \mathrm{s}$ [43]. Thus, it is quite feasible to successfully implement the proposed MAS with the above mentioned delays considering modern communication facility of the system.

\section{CONCLUSION}

Within the structure of modern power system control, a multi-agent based emergency control scheme under multiple contingencies has been proposed in this paper. The simulation results show the effectiveness of the proposed control strategy to maintain acceptable voltage profile under emergency conditions. This method can provide quick and effective voltage support in system contingencies when the disturbances in the affected zone can be identified. However, it is necessary to facilitate interaction among the neighboring zones when more than one zone is taking countermeasures to account for the effect of the overall control action. The main contribution of the paper is the novel adaptive determination of the local zones and the development of a multi-agent decentralized control algorithm to determine the most optimum countermeasures at zones near the disturbances to maintain the load voltages and reactive power outputs of the generators in the allowable operating limits.

\section{REFERENCES}

[1] W.R. Lachs, "A new horizon for system protection schemes," IEEE Trans. on Power Systems, vol.18, no.1, pp.334,338, Feb 2003

[2] P. Hines, J. Apt, S. Talukdar, "Large blackouts in North America: Historical trends and policy implications”, Energy Policy, Vol. 37, Issue 12, pp. 5249-5269, December 2009.

[3] US-Canada Power System Outage Task Force, "Final Report on the August 14, 2003 Blackout in the United States and Canada: Causes and Recommendations", [Online], Available Nov. 2013: http://certs.lbl.gov/pdf/b-f-web-part1.pdf

[4] UCTE Report, "Final Report: System Disturbance on 4 November, 2006”, [Online], Available Nov. 2013:

http://www.geocities.jp/ps_dictionary/blackout/Final-Report20070130.pdf

[5] Department of Primary Industries, "16th January 2007 electricity supply interruptions in Victoria - What happened, why, opportunities and recommendations”, July 2007, The Nous group publication, www.nousgroup.com.au, pages $1-24$.

[6] "Report of the Enquiry Committee on Grid Disturbance in Northern Region on 30th July 2012 and in Northern, Eastern \& North-Eastern Region on 31st July 2012”, http://www.powermin.nic.in Retrieved 28 August 2012.

[7] V.C. Nikolaidis, C.D. Vournas, "Design Strategies for Load-Shedding Schemes Against Voltage Collapse in the Hellenic System,” IEEE Trans. on Power Systems, vol.23, no.2, pp.582,591, May 2008

[8] W. R. Lachs and Sutanto, D., "Voltage Instability in Interconnected Power Systems: a Simulation Approach," IEEE Trans. on Power Systems, vol.7, no.2, pp.753-761, May 1992.

[9] W. R. Lachs, "Controlling grid integrity after power system emergencies," Power Systems, IEEE Transactions on, vol.17, no.2, pp.445-450, May 2002

[10] S. D. J. McArthur, E. M. Davidson, V. M. Catterson, A. L. Dimeas, N. D. Hatziargyriou, F. Ponci and T. Funabashi, "Multi-Agent Systems for Power Engineering Applications-Part I: Concepts, Approaches, and Technical Challenges,” IEEE Trans. on Power Systems, vol.22, no.4, pp.1743-1752, Nov. 2007.
[11] H. S. V. S. Kumar Nunna and and Suryanarayana Doolla, "Energy Management in Microgrids Using Demand Response and Distributed Storage-A Multiagent Approach", IEEE Trans. on Power Delivery, vol. 28, no. 2, pp. 939-947, April 2013

[12] Su Sheng, K. K. Li, W.L. Chan, Xiangjun Zeng, Dongyuan Shi and Xianzhong Duan, "Adaptive Agent-Based Wide-Area Current Differential Protection System,” IEEE Trans. on Industry Applications, vol.46, no.5, pp.2111,2117, Sept.-Oct. 2010

[13] T. Logenthiran, D. Srinivasan, A.M. Khambadkone and Htay Nwe Aung, "Multiagent System for Real-Time Operation of a Microgrid in Real-Time Digital Simulator," IEEE Transactions on Smart Grid, vol.3, no.2, pp.925,933, June 2012

[14] M. Negnevitsky, N. Voropai, V. Kurbatsky, N. Tomin and D. Panasetsky, "Development of an intelligent system for preventing largescale emergencies in power systems," IEEE Power and Energy Society General Meeting (PES), 2013 , pp.1-5, 21-25 July 2013

[15] F. Ren, M. Zhang, D. Sutanto, X. D. Su, "Conceptual Design of a Multi Agent System for Interconnected Power Systems Restoration,” IEEE Trans. on Power Systems, vol.27, no.2, pp.732-740, 2012.

[16] H. F. Wang, H. Li and H. Chen, "Coordinated Secondary Voltage Control to Eliminate Voltage Violations in Power System Contingencies,” IEEE Trans. on Power Systems, vol.18, no.2, pp.588595, May 2003.

[17] M. R. Tousi, S. H. Hosseinian and M. B. Menhaj, “A Multi-Agent based Voltage Control in Power System Using Distributed Reinforcement Learning”, Journal Simulation, vol.87, no.7, pp.581-599, July 2011.

[18] G. Sheng, X. Jiang, D. Duan and G. Tu, "Framework and Implementation of Secondary Voltage Regulation Strategy based on Multi-Agent Technology”, Int. Journal of Electrical Power and Energy Systems, vol.31, no.1, pp.67-77, January 2009.

[19] M. Ishida, T. Nagata, H. Saiki, I. Shimada and R. Hatano, "A MultiAgent based Cooperative Voltage and Reactive Power Control", Electrical Engineering in Japan, vol.181, no.2, 2012.

[20] D. A. Panasetsky and N. I. Voropai, "A Multi-Agent Approach to Coordination of Different Emergency Control Devices against Voltage Collapse," in Proc. 2009 IEEE Bucharest PowerTech, pp.1-7, June 28 July 22009.

[21] M.E. Baran and I.M. El-Markabi, "A Multiagent-Based Dispatching Scheme for Distributed Generators for Voltage Support on Distribution Feeders,” IEEE Trans. on Power Systems, vol.22, no.1, pp.52,59, Feb. 2007

[22] P. Lagonotte, J. C. Sabonnadiere, J. Y. Leost, and J. P. Paul, "Structural Analysis of the Electrical System: Application to Secondary Voltage Control in France," IEEE Trans. on Power Systems, vol.4, no.2, pp.479485, May 1989.

[23] L. Hang, A. Bose and V. Venkatasubramanian, "A Fast Voltage Security Assessment Method using Adaptive Bounding." IEEE Trans. on Power Systems, vol.15, no.3, pp.1137-1141, Aug 2000.

[24] J. Zhong, E. Nobile, A. Bose and K. Bhattacharya. "Localized Reactive Power Markets using the Concept of Voltage Control Areas." IEEE Trans. on Power Systems, vol.19, no.3, pp.1555-1561, Aug. 2004.

[25] H Liu; H. Bao; L. Liu; , "A new method about calculating electrical distance," Power Engineering and Automation Conference (PEAM), 2011 IEEE , vol.1, no., pp.382-385, 8-9 Sept. 2011

[26] S. Blumsack, P. Hines, M. Patel, C. Barrows and E. C. Sanchez, "Defining Power Network Zones from Measures of Electrical Distance," in Proc. IEEE Power \& Energy Society General Meeting, 2009, pp.1-8, 26-30 July, 2009.

[27] J. A. Hartigan, and M. A. Wong, "A K-Means Clustering Algorithm," Applied Statistics, Vol.28, No.1, pp. 100-108.

[28] Feng Dong, Badrul H. Chowdhury, Mariesa L. Crow and Levent Acar, "Improving Voltage Stability by Reactive Power Reserve Management" IEEE Trans. On Power Systs., vol. 20, no. 1, February 2005

[29] Ian Dobson and Liming Lu, "Voltage Collapse Precipitated by the Immediate Change in Stability When Generator Reactive Power Limits are Encountered" IEEE Trans. on Circuits And Systems-I: Fundamental Theory And Applications, Vol. 39, No. 9, September 1992.

[30] M. Begovic and A. Phadke, "Control of voltage stabilityusing sensitivity analysis”, IEEE Trans. On Power Systems, vol. 7, no. 1, pp.114 -123 1992

[31] B. Stott and O. Alsac, "Fast Decoupled Load Flow," IEEE Trans. on Power Apparatus and Systems, vol.PAS-93, no.3, pp.859-869, 1974.

[32] T. Van Cutsem, C.D. Vournas, "Emergency Voltage Stability Controls: an Overview," in proc. IEEE Power Engineering Society General Meeting Tampa, FL, 2007, p. 1. 
[33] D. Choi, S. Lee, D. Won and S. Kim, "Efficient Secure Group Communications for SCADA,” IEEE Trans. on Power Delivery, vol.25, no.2, pp.714-722, April 2010.

[34] F. Bellifemine, G. Caire, and D. Greenwood, "Developing Multi-Agent Systems with JADE”, vol. 5. Wiley, 2007.

[35] S. Russell and P. Norvig, Artificial Intelligence: A Modern Approach. Englewood Cliffs, NJ: Prentice Hall, 1995.

[36] Greg O'Hare and N. R. Jennings, Foundation of Distributed Artificial Intelligence. New York: Wiley, 1996, pp. 4-46, ISBN 0-471-00675-0.

[37] Y. Jihoon and R. Havaldar, "Coordination of Distributed Knowledge Networks using Contract Net Protocol,” in Proc. IEEE Information Technology Conf., 1998, pp.71-74.

[38] "Power System Test Case Archive”, University of Washington, College of Engineering, Electrical Engineering, 2008. [Online]. Available Nov. 2013: http://www.ee.washington.edu/research/pstca/
[39] F. Milano, “An Open Source Power System Analysis Toolbox,” IEEE Trans. on Power Systems, vol. 20, no. 3. pp. 1199-1206, Aug. 2005.

[40] "Instrument Control Toolbox". [Online]. Available: http://www.mathworks.com/products/instrument/

[41] J. Mozina "Undervoltage load shedding-Part 2", Electric Energy T\&D Mag., pp.26 -32 2006

[42] X. Tong, X. Wang, R. Wang, F. Huang, X. Dong, K.M. Hopkinson, G. Song, "The Study of a Regional Decentralized Peer-to-Peer NegotiationBased Wide-Area Backup Protection Multi-Agent System," IEEE Transactions on Smart Grid, , vol.4, no.2, pp.1197,1206, June 2013C.

[43] T. Skeie, S. Johannessen, and C. Brunner, "Ethernet in substation automation,”IEEE Control Syst. Mag., vol. 22, no. 3, pp. 43-51, Jun. 2002. 